\title{
Individualized computer-based surgical planning to address pulmonary arteriovenous malformations in patients with a single ventricle with an interrupted inferior vena cava and azygous continuation
}

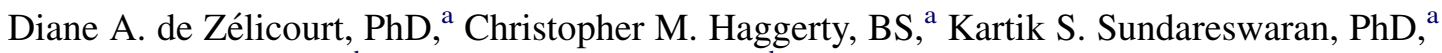 \\ Brian S. Whited, PhD, ${ }^{\mathrm{b}}$ Jarek R. Rossignac, PhD, ${ }^{\mathrm{b}}$ Kirk R. Kanter, MD, ${ }^{\mathrm{c}} \mathrm{J}$. William Gaynor, MD, ${ }^{\mathrm{d}}$ \\ Thomas L. Spray, MD, ${ }^{\mathrm{d}}$ Fotis Sotiropoulos, PhD, ${ }^{\mathrm{e}}$ Mark A. Fogel, MD, ${ }^{\mathrm{f}}$ and Ajit P. Yoganathan, $\mathrm{PhD}^{\mathrm{a}}$
}

\begin{abstract}
Objective: Pulmonary arteriovenous malformations caused by abnormal hepatic flow distribution can develop in patients with a single ventricle with an interrupted inferior vena cava. However, preoperatively determining the hepatic baffle design that optimizes hepatic flow distribution is far from trivial. The current study combines virtual surgery and numeric simulations to identify potential surgical strategies for patients with an interrupted inferior vena cava.
\end{abstract}

\begin{abstract}
Methods: Five patients with an interrupted inferior vena cava and severe pulmonary arteriovenous malformations were enrolled. Their in vivo anatomies were reconstructed from magnetic resonance imaging $(n=4)$ and computed tomography $(\mathrm{n}=1)$, and alternate virtual surgery options (intracardiac/extracardiac, Y-grafts, hepatoto-azygous shunts, and azygous-to-hepatic shunts) were generated for each. Hepatic flow distribution was assessed for all options using a fully validated computational flow solver.
\end{abstract}

\begin{abstract}
Results: For patients with a single superior vena cava $(n=3)$, intracardiac/extracardiac connections proved dangerous, because even a small left or right offset led to a highly preferential hepatic flow distribution to the associated lung. The best results were obtained with either a Y-graft spanning the Kawashima to split the flow or hepato-to-azygous shunts to promote mixing. For patients with bilateral superior vena cavae $(\mathrm{n}=2)$, results depended on the balance between the left and right superior inflows. When those were equal, connecting the hepatic baffle between the superior vena cavae performed well, but other options should be pursued otherwise.
\end{abstract}

Conclusions: This study demonstrates how virtual surgery environments can benefit the clinical community, especially for patients with a single ventricle with an interrupted inferior vena cava. Furthermore, the sensitivity of the optimal baffle design to the superior inflows underscores the need to characterize both preoperative anatomy and flows to identify the best option. (J Thorac Cardiovasc Surg 2011;141:1170-7)

Pulmonary arteriovenous malformations (PAVMs) are an uncommon but lethal complication after the superior cavopulmonary palliation of single ventricle heart diseases,

From the Department of Biomedical Engineering, , Georgia Institute of Technology,

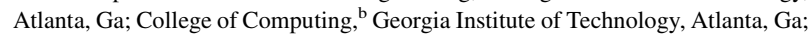
Division of Cardiothoracic Surgery, ${ }^{\mathrm{c}}$ Emory University School of Medicine, Atlanta, Ga; Division of Cardiology, ${ }^{\mathrm{d}}$ Children's Hospital of Philadelphia, Philadelphia, Pa; St Anthony Falls Laboratory, ${ }^{\mathrm{e}}$ University of Minnesota, Minneapolis, Minn; and Division of Cardiothoracic Surgery, ${ }^{\mathrm{f}}$ Children's Hospital of Philadelphia, Philadelphia, Pa.

This study was supported by the National Heart, Lung, and Blood Institute Grants HL-67622 and HL-098252.

Disclosures: Authors have nothing to disclose with regard to commercial support.

Read at the 36th Annual Meeting of The Western Thoracic Surgical Association, Ojai, California, June 23-26, 2010.

Received for publication July 27, 2010; revisions received Nov 5, 2010; accepted for publication Nov 23, 2010; available ahead of print Feb 21, 2011.

Address for reprints: Ajit P. Yoganathan, PhD, Wallace H. Coulter School of Biomedical Engineering, Georgia Institute of Technology and Emory University, 313 Ferst Drive, Atlanta, GA 30332-0535 (E-mail: ajit.yoganathan@bme.gatech.edus). 0022-5223/\$36.00

Copyright (C) 2011 by The American Association for Thoracic Surgery doi: $10.1016 /$ j.jtcvs.2010.11.032 which are characterized by the appearance of intrapulmonary arterial-to-venous shunts that bypass the pulmonary gas exchange units and result in a net decrease in oxygen saturation. These shunts lead to a decrease in pulmonary vascular resistance, thereby directing more flow to the diseased lung, and can create a positive feedback loop of increasing hypoxemia. One single ventricle subgroup that is especially at risk for PAVMs is children with an interrupted inferior vena cava (IVC) and azygous (AZ) continuation, for whom PAVM occurrence can be as high as $18 \%$ to $21 \%$ after the Kawashima procedure. ${ }^{1}$ Although the underlying mechanism leading to PAVMs is unknown, studies have shown that liver-derived factors present in the hepatic venous blood prevent the formation of PAVMs. ${ }^{1-4}$ Following the Kawashima procedure with the completion of the total cavopulmonary connection (TCPC) was thus suggested as an efficient mean to restore hepatic flow to the lungs and resolve PAVMs. ${ }^{5}$

The TCPC does not completely eliminate the risk of PAVM development. Poor design of the IVC and hepatic 

Abbreviations and Acronyms
$\mathrm{AZ}=$ azygous
$\mathrm{CO}=$ cardiac output
HepV $=$ hepatic veins
HFD = hepatic flow distribution
IV = innominate vein
IVC = inferior vena cava
LPA = left pulmonary artery
LSVC = left superior vena cava
PA = pulmonary artery
PAVM = pulmonary arteriovenous malformation
RPA = right pulmonary artery
SVC = superior vena cava
$\mathrm{TCPC}=$ total cavopulmonary connection

venous baffle may lead to an unbalanced hepatic flow distribution (HFD) to the left and right lungs, and in turn to unilateral PAVMs. As discussed by Sundareswaran and colleagues, ${ }^{6}$ the low flow rate coming through the hepatic baffle in patients with an interrupted IVC (which accounts for only $\sim 20 \%-25 \%$ of the systemic venous return, as opposed to the normal $50 \%-60 \%$ carried by the IVC) increases the complexity of the flow interactions at the center of the TCPC, which in turn increases the difficulty of identifying the best surgical approach for a specific patient on the basis of anatomic considerations alone. Thus, patients with an interrupted IVC remain at greater risk than other single ventricle cases for the development of PAVM, even with a completed TCPC. Once the extent of PAVMs is such that oxygen saturation is critically low, the only palliative option is to reoperate and reorient the hepatic baffle to achieve a better HFD. ${ }^{1}$

Our group recently presented a novel surgical planning framework ${ }^{6}$ that enables surgeons to virtually perform multiple surgical scenarios and determine the one that will yield the best hemodynamic performance before even entering the operating room. Such a surgical planning platform offers a unique solution for cases such as these, in which hemodynamics (and in particular HFD) are vital to the surgery, but in which the small patient population and large number of anatomic variations pose a severe obstacle to the establishment of surgical guidelines from clinical studies alone.

This study reviews our experience in the planning and optimization of the Fontan surgery to characterize the options available to surgeons to prevent or revert PAVMs in patients with a single ventricle with an interrupted IVC. Although based on a small sample size, the systematic review of the TCPC variations investigated for each patient sheds light on the impact of caval offset and other design parameters on TCPC hemodynamics, and allows for a better understanding of the anatomy- or flow-based character- izations that can be made for the definition of "global" surgical strategies.

\section{MATERIALS AND METHODS \\ Patient Data}

Five patients with a single ventricle with an interrupted IVC and severe PAVMs were enrolled at the Children's Hospital of Philadelphia or Children's Healthcare of Atlanta for surgical planning at the Georgia Institute of Technology. The study was approved by the institutional review boards of all institutions. Patients were separated into 2 groups according to the configuration of the superior venous returns. Group A, or the single SVC group, included the 3 patients in whom the superior vena cava (SVC), $\mathrm{AZ}$, and innominate vein (IV) merged together at or before the pulmonary arteries (PAs). Group B included the 2 patients in whom the IV did not adjoin the SVC but connected directly to the PAs in the form of a persistent left SVC (LSVC). PAVMs were diagnosed by angiographic appearance and pulse oximetry $(72 \% \pm 4 \%$ at the time of referral). Table 1 summarizes the clinical, anatomic, and flow information relevant to this study. Patients A1, $\mathrm{A} 2$, and B2 had a completed TCPC and a unilateral PAVM diagnosis. Patient A3 was still at the Kawashima stage. Patient B1 had previously undergone a TCPC that had been taken down because of a clot in the hepatic baffle. Both patients A3 and B1 had a bilateral PAVM diagnosis.

\section{Methods}

The pillars of the framework in this study were clinical imaging and volume rendering of the in vivo anatomy and flow, virtual surgery, and performance quantification using numeric modeling.

Clinical imaging. Most patients underwent a cardiac magnetic resonance imaging evaluation, including an anatomic dataset of the entire thorax to reconstruct the cardiovascular geometry ${ }^{7}$ and through-plane velocity maps acquired across all inflows (AZ, IV, SVC, hepatic veins [HepV]) and outflows (left PA [LPA] and right PA [RPA]) for flow boundary conditions. Patient A2 had coils and pacer wires that prevented exposure to magnetic radiations; therefore, anatomic information for that patient was obtained by x-ray computed tomography. The reconstructed anatomies of all 5 patients are shown in Figure 1. To compensate for the lack of flow data from computed tomography for patient A2, inflow conditions were imposed using the inflow conditions of patient A1, who had a fairly similar body surface area and age. The corresponding flow information is noted with an asterisk in Table 1 to be easily differentiated from the patient-specific flow measurements.

Virtual surgery. Multiple corrective anatomies were generated for each patient using a novel virtual surgery interface. ${ }^{8}$ These surgical options (including intra/extracardiac grafts, bifurcated Y-shaped grafts, HepV-to$\mathrm{AZ}$, and AZ-to-HepV shunts) were carefully selected on the basis of the currently available surgical techniques and the "available space" around the connection. The geometric constraints imposed by the surrounding organs were accounted for by including the heart and great vessels into the interface, as shown in Figure 1 for patients A3 and B1.

Performance quantification. Numeric simulations were conducted on all envisioned options using an in-house unstructured immersed-boundary method ${ }^{9}$ to model the complex anatomic flows with elevated spatial resolution. Inflow boundary conditions were prescribed using the in vivo magnetic resonance imaging flow rates averaged over the cardiac cycle. Outflows, on the other hand, tightly depend on the surgical design retained. If an option successfully increases HFD to the diseased lung, PAVMs will regress, increasing the lung resistance on that side and subsequently decreasing the flow to that lung. Accordingly, each surgical option was tested over a wide range of LPA/RPA flow ratios to best predict HFD (and ultimately PAVM) evolution. HFD was assessed as a postprocessing by uniformly seeding weightless point particles across the HepV cross-section and quantifying the flux of hepatic particles exiting through the LPA and RPA, respectively. 
TABLE 1. Summary of the clinical and flow characteristics of the five patients enrolled in the study

\begin{tabular}{|c|c|c|c|c|c|}
\hline & \multicolumn{3}{|c|}{ Group A: single SVC } & \multicolumn{2}{|c|}{ Group B: persistent LSVC } \\
\hline & A1 & A2 & $\mathbf{A 3}$ & B1 & B2 \\
\hline Gender & Female & Male & Female & Female & Male \\
\hline Age (y) & 4 & 6 & 3 & 4 & 11 \\
\hline $\operatorname{BSA}\left(\mathrm{m}^{2}\right)$ & 0.61 & 0.75 & 0.635 & 0.64 & 1.22 \\
\hline TCPC stage & EC TCPC & IA TCPC & Kawashima & $\begin{array}{l}\text { Kawashima } \\
\text { (TCPC taken down) }\end{array}$ & EC TCPC \\
\hline \multicolumn{6}{|l|}{ Systemic venous return } \\
\hline $\mathrm{Q}_{\mathrm{s}}$ & $3.43 \mathrm{~L} / \mathrm{min}$ & $3.43 \mathrm{~L} / \mathrm{min}^{*}$ & $3.86 \mathrm{~L} / \mathrm{min}$ & $2.93 \mathrm{~L} / \mathrm{min}$ & $3.95 \mathrm{~L} / \mathrm{min}$ \\
\hline $\mathrm{Q}_{\mathrm{HepV}}\left(\% Q_{S}\right)$ & $21 \%$ & $21 \% *$ & $32 \%$ & $21 \%$ & $10 \%$ \\
\hline $\mathrm{Q}_{\mathrm{AZ}}\left(\% Q_{S}\right)$ & $24 \%$ & $24 \% *$ & $17 \%$ & $15 \%$ & $30 \%$ \\
\hline $\mathrm{Q}_{\mathrm{svC}}\left(\% Q_{S}\right)$ & $33 \%$ & $33 \% *$ & $51 \%$ & $\mathrm{n} / \mathrm{a}$ & $\mathrm{n} / \mathrm{a}$ \\
\hline $\mathrm{Q}_{\mathrm{IV}}\left(\% Q_{S}\right)$ & $22 \%$ & $22 \% *$ & & $\mathrm{n} / \mathrm{a}$ & $\mathrm{n} / \mathrm{a}$ \\
\hline $\mathrm{Q}_{\mathrm{RSVC}}\left(\% Q_{S}\right)$ & $\mathrm{n} / \mathrm{a}$ & $\mathrm{n} / \mathrm{a}$ & $\mathrm{n} / \mathrm{a}$ & $38 \%$ & $40 \%$ \\
\hline $\mathrm{Q}_{\mathrm{LSVC}}\left(\% Q_{S}\right)$ & $\mathrm{n} / \mathrm{a}$ & $\mathrm{n} / \mathrm{a}$ & $\mathrm{n} / \mathrm{a}$ & $26 \%$ & $20 \%$ \\
\hline PAVM location & Left & Right & Bilateral & Bilateral & Right \\
\hline \multicolumn{6}{|l|}{ Oxygen saturation } \\
\hline Preoperative & $72 \%$ & $67 \%$ & $75 \%$ & $76 \%$ & $70 \%$ \\
\hline $\begin{array}{l}\text { Postoperative } \\
\text { (follow-up time) }\end{array}$ & $94 \%(5 \mathrm{mo})$ & $80 \%(<1 \mathrm{mo})$ & $97 \%(15.5 \mathrm{mo})$ & $92 \%(25 \mathrm{mo})$ & $87 \%(4.5 \mathrm{mo})$ \\
\hline
\end{tabular}

Group A: Single SVC

\begin{tabular}{ccc}
\hline (A1) & (A2) & (A3) \\
Left PAVM & Right PAVM & Bilateral PAVM \\
\hline
\end{tabular}
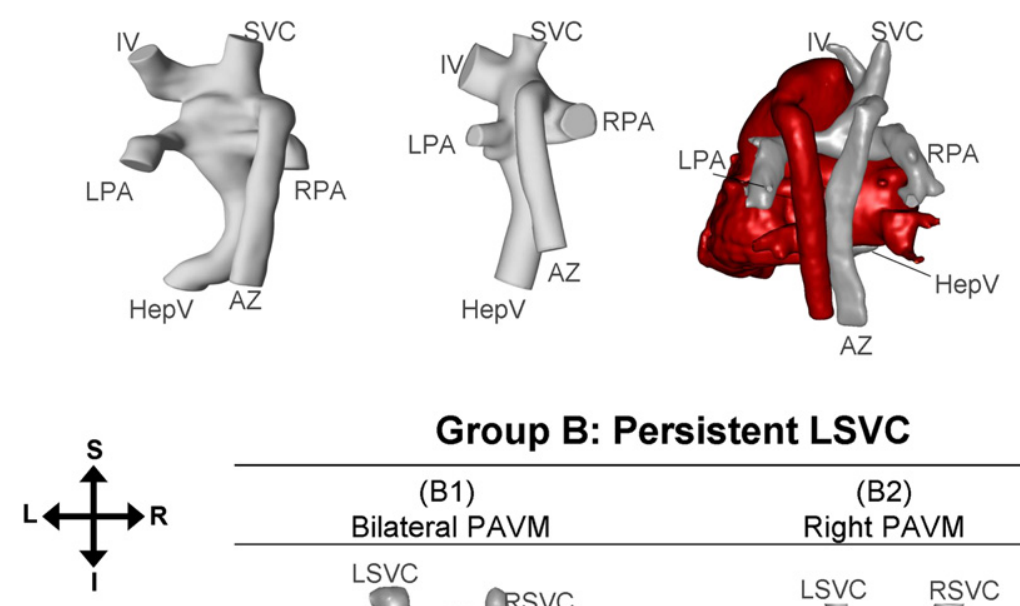

Group B: Persistent LSVC

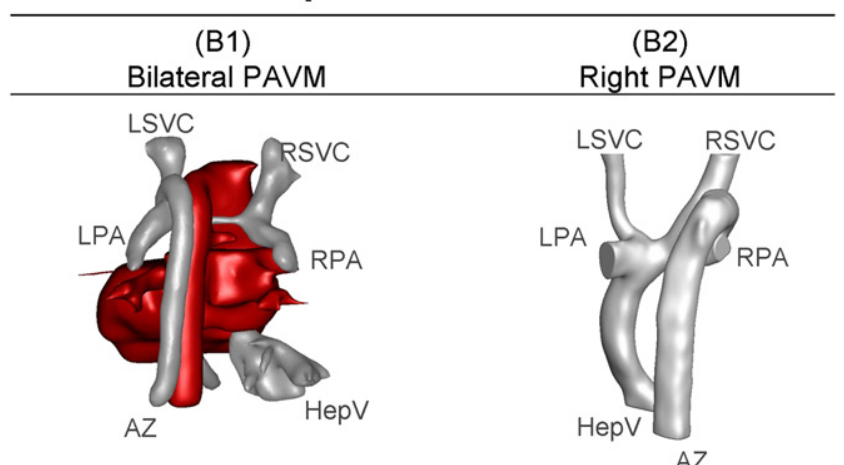

FIGURE 1. Patient anatomies seen from the posterior side. The heart is shown in red for the 2 patients without a completed TCPC to illustrate the constraints imposed by the surrounding vascular structures. Orientation axis: $\mathrm{S}=$ superior; $\mathrm{I}=$ inferior; $\mathrm{L}=$ left; $\mathrm{R}=$ right. $S V C$, Superior vena cava; $P A V M$, pulmonary arteriovenous malformation; $I V$, innominate vein; $L P A$, left pulmonary artery; $R P A$, right pulmonary artery; $A Z$, azygous; $H e p V$, hepatic veins; $L S V C$, left superior vena cava. 


\section{RESULTS}

\section{Group A: Patients With Single Superior Vena Cava}

Figure 2 shows selected options generated for the patients with a single SVC, along with the achieved HFD. The superimposed black arrows show the main flow direction of the superior venous returns, and the dashed lines denote the axis of the PAs and hepatic baffle. The HFD is qualitatively represented by the red and blue stream traces and quantified in percent under each option. Options yielding an HFD that closely follows the global flow distribution are highlighted in green, as potential candidates for the surgery, at least in terms of HFD performance.

The effect of different anastomosis, or offset, locations between the hepatic baffle and the Kawashima connection were compared (Figure 2, $A$ and $B$ ). As shown in Figure 2, $A$, any offset to the left of the Kawashima connection resulted in an almost uni-sided HFD to the left lung, with more than $90 \%$ of the flow going to the LPA for all 3 patients. A symmetric behavior was observed when shifting the hepatic baffle to the right of the Kawashima connection, resulting in an almost uni-sided HFD to the RPA. Such offset configuration corresponds to what had originally been implemented for patient A2 (Figure 2, A) and had led to severe PAVMs in the contralateral lung, which is in line with previ- ous literature relating PAVMs to a low supply in hepatic nutrients. $^{1-4}$

Figure 2, $B$, shows the results obtained when seeking to avoid any offset and aiming for the geometric center of the connection in an effort to improve mixing between the superior inflows and the hepatic blood flow. This approach is only successful for patient A3, who had the highest hepatic flow rate and in whom the superior and hepatic flows effectively collide in a head-on fashion. For patients A1 and A2, on the other hand, the AZ flow slightly skews the superior inflows toward the LPA, such that the hepatic flow enters the connection to the right of the superior inflows, resulting in a highly biased HFD to the RPA. Aiming for the geometric center of the connection thus does not warrant an adequate HFD; rather, it is the position of the baffle relative to the center of the superior bloodstream that seems to be key in achieving the desired HFD. However, as is illustrated by patients A1 and A2, this "flow center" is difficult to precisely locate on the basis of anatomic considerations alone and may evolve with time depending on the configuration of the superior vessels and their relative contribution to the systemic venous return.

To circumvent the difficulties posed by the definition of an optimal offset relative to the "flow center," 3 other

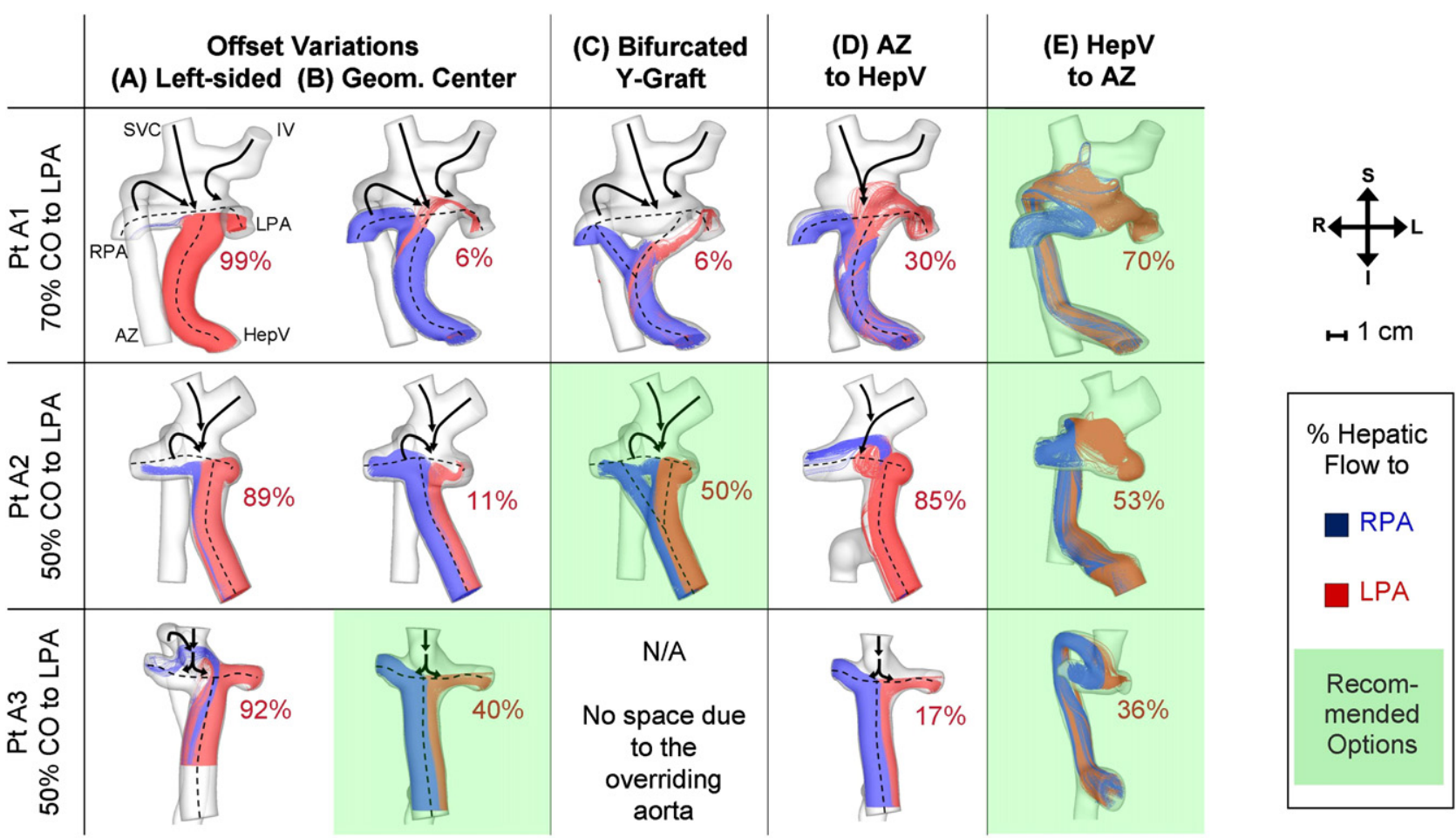

FIGURE 2. HFD for selected TCPC options for the 3 patients with a single SVC, including intra-atrial or extracardiac options with (A) an offset to the LPA or (B) aiming to the center of the Kawashima connection, (C) bifurcated Y-grafts, (D) intra-atrial or extracardiac options combined with an AZ-to-HepV shunt, and (E) HepV-to-AZ shunts. Percentages indicate HFD to the left lung. Superimposed black arrows show the main flow direction of the superior inflows. Dashed lines denote vessel axes. Orientation axis: $\mathrm{S}=$ superior; $\mathrm{I}=$ inferior; $\mathrm{L}=$ left; $\mathrm{R}=$ right. AZ, Azygous; HepV, hepatic veins; N/A, not available; $C O$, cardiac output; $L P A$, left pulmonary artery; $R P A$, right pulmonary artery. 
options were attempted: (1) dividing the hepatic baffle into 2 branches, reaching to the left and right of the Kawashima connection (Y-graft); (2) merging the AZ flow into the hepatic baffle to increase the momentum of the flow coming through the baffle and increase mixing at the center of the connection (AZ-to-HepV); or (3) routing the hepatic flow to the $A Z$ vein to force the mixing of all venous returns in the Kawashima connection (HepV-to-AZ). The Y-graft for patient A2 was successful because the left and right branches spanned the Kawashima connection, and the graft bifurcation was designed with a relatively small angle to allow a proper splitting of the hepatic flow into 2 branches. For patient A1, the overriding aorta did not allow the left branch of the graft to reach beyond the Kawashima connection, resulting in poor HFD performance, similar to the preoperative "no offset" configuration. Similarly, the overriding aorta prevented the implementation of a bifurcated graft for patient A3. It is noteworthy that these anatomic constraints were circumvented using an intra-atrial approach for the left branch in patient A2.

AZ-to-HepV shunts (Figure 2, D) decrease the sensitivity to offset, increasing mixing at the center of the connection, as demonstrated by the hepatic stream traces that penetrate deeper into the connection area than without the shunt. However, this procedure was not the best for any of the patients, partly because the AZ-to-HepV shunts were designed in combination with the existing preoperative baffles to minimize surgical complexity. Better performances may have been obtained by modifying the baffle offset or performing surgical planning before the first surgery to optimize the original baffle with combined $\mathrm{AZ}$ and HepV flows.

Connecting the HepV to the AZ (Figure 2, E) was established as a suitable option in terms of HFD for all 3 patients. Because that option produced thorough mixing of all inflows, HFD was found to closely follow the global cardiac output (CO) distribution. In patient A3, in particular, the Hepto-AZ graft led to a $36 \%$ HFD to the left lung when $50 \%$ $\mathrm{CO}$ went to that lung. On average, HFD to the left lung scaled as $(0.9 \pm 0.2) * \mathrm{CO}$ to the LPA. However, that option also increased energy expenditure because of the increased viscous dissipation in the AZ. This issue may become prevalent in young patients with small vasculature in whom small AZ dimensions may lead to elevated power losses.

The surgical approaches recommended for the 3 patients with a single SVC were a Y-graft for patient A2; a no-offset intra-atrial graft for patient $\mathrm{A} 3$, because those combined both HFD and energy efficiency; and an HepV-to-AZ shunt for patient A1, setting the priority on optimizing HFD even at a higher energy expenditure.

\section{Group B: Patients With Bilateral Superior Vena Cavae}

Figure 3 shows the HFD performance associated with selected surgical planning options envisioned for patient B1. The impact of offset variations is shown in Figure $3, A-C$. For patients with a single SVC, small changes in the baffle anastomosis location relative to the SVC led to large
(A) To RPA

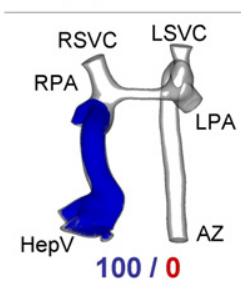

\section{Offset Variations}

(B) To RSVC

(C) To mid-PA

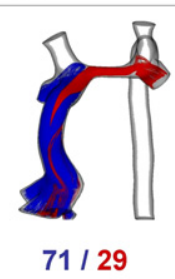

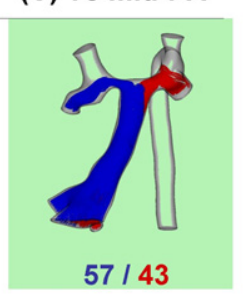

$57 / 43$

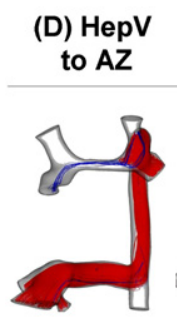

$4 / 96$
(E) AZ to HepV

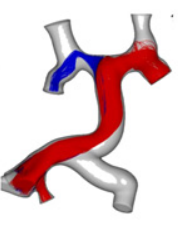

$20 / 80$
(F) Y-Graft

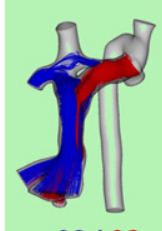

$62 / 38$
Split Hepatic Flow

(G) H-connection

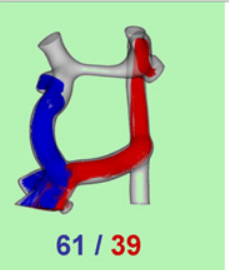

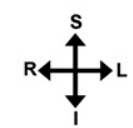

$\rightarrow 1 \mathrm{~cm}$

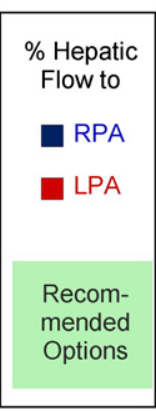

FIGURE 3. HFD for selected TCPC options for patient B1, under a global CO distribution of 50/50 RPA/LPA. Options include extracardiacs and intraatrials with (A-C) an offset shifting from the RPA to the mid-PA; (D) a HepV-to-AZ shunt; an (E) AZ-to-HepV shunt connected to the mid-PA; and 2 options seeking to divide the hepatic flow, (F) a Y-graft and (G) an H-connection. HFD to the right and left lungs is indicated below the figures (in blue and red, respectively). Orientation axis: $\mathrm{S}=$ superior; $\mathrm{I}=$ inferior; $\mathrm{L}=$ left; $\mathrm{R}=$ right. $A Z$, Azygous; HepV, hepatic veins; N/A, not available; CO, cardiac output; $L P A$, left pulmonary artery; $R P A$, right pulmonary artery. 
variations in HFD. For patients with a single SVC, an option that seemed to work systematically was to circumvent the problem altogether using an HepV-to-AZ shunt. However, as shown in Figure 3, D, this approach fails for patients with a bilateral SVC, resulting in a configuration similar to a strong offset toward the LPA (or the RPA if the AZ was connected to the RSVC instead of the LSVC) with a uni-sided HFD, which may ultimately favor PAVMs in the contralateral lung.

On the other hand, although the most challenging question for the single SVC cases was the location of the center of the superior inflows, the separation of the superior inflows in patients with bilateral SVC (with the RSVC on the right and the AZ and LSVC on the left) might provide an easier answer to that question, with the mid-PA acting as the ideal "no offset" location. For patient B1, for example, the RSVC flow rate $(1.1 \mathrm{~L} / \mathrm{min})$ almost exactly matched the LSVC and AZ flows combined $(1.2 \mathrm{~L} / \mathrm{min})$. As a result, when the baffle was connected to the mid-PA segment as in Figure 3, $C$, the RSVC flows to the RPA, the AZ+LSVC flows to the LPA, and the hepatic flow adjusted its distribution between the LPA and the RPA to achieve the desired global flow distribution, resulting in an HFD of 57/43 RPA/LPA for a global flow distribution of 50/50. The 2 other positive approaches in terms of HFD were to divide the hepatic flow into 2 branches, using a Y-graft (Figure $3, F$ ) or an $\mathrm{H}$-connection combining an extracardiac graft offset toward the RPA and an HepV-to-AZ shunt to reach the LPA (Figure 3, G), both of which had approximately the same effect as the mid-PA connection.

Patient B2 represents the most challenging anatomic and flow configurations encountered in the course of this study. As shown in Figure 1, the RSVC attached to the PAs close to the LSVC anastomosis site, such that the mid-PA segment was not well defined. Furthermore, the LSVC accounted for only $20 \%$ of the systemic venous return, whereas the AZ and RSVC combined accounted for $70 \%$, yielding highly unbalanced left and right contributions. Finally, the hepatic blood flow represented only $10 \%$ of the CO.

Given the extremely low hepatic flow rate, HFD for patient B2 was extremely sensitive to the TCPC design and the competition with the superior inflows. Figure 4 shows the flow patterns and HFD observed in 4 selected options for patient B2. The detrimental consequence of the extremely low hepatic flow rate is apparent in Figure 4, A and $B$, where the RSVC does not encounter any resistance from the hepatic blood flow, penetrates deep into the baffle, and forces the hepatic flow toward the LPA, thereby preventing any hepatic flow to the RPA. After investigating a number of alternate options, the 2 best approaches were to perform an $\mathrm{H}$-connection using the preoperative extracardiac graft and an HepV-to-AZ shunt (Figure 4, C), or to use a Y-graft in combination with an AZ-to-HepV shunt (Figure 4, D).

On the basis of the results obtained for patient B1, the mid-PA connection may seem to be an attractive "by default" option, all the more because parametric variations revealed that the HFD did not depend on the type of graft retained (extracardiac or intra-atrial) or the exact anastomosis location as long as the graft connected somewhere between the RSVC and the LSVC. However, it should be emphasized that the success of this approach for patient B1 relied on the 3 following conditions: (1) The mid-PA segment was well defined, preventing any interaction between the left and right superior venous return, and allowing
(A) Intra-atrial
(B) Y-Graft
(C) H-connection

\section{(D) AZ-to-HepV} to RSVC and Y-Graft

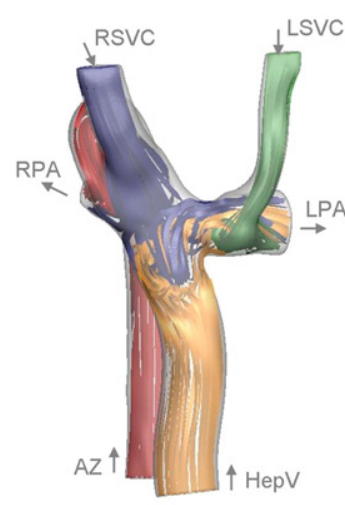

$0 / 100$

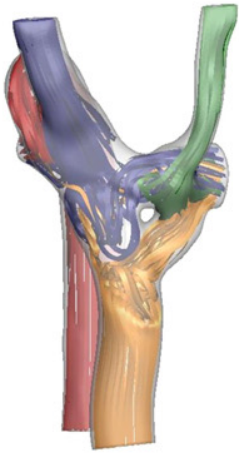

$0 / 100$

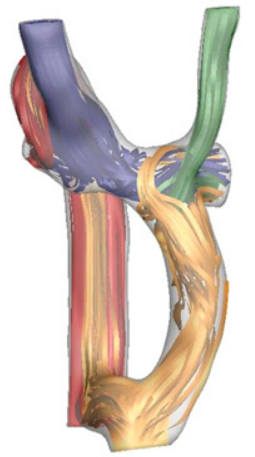

$71 / 29$

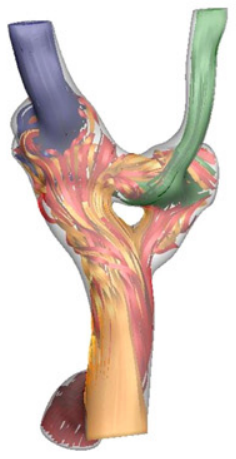

$34 / 66$

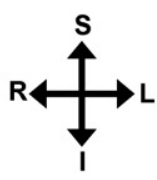

$\mapsto 1 \mathrm{~cm}$

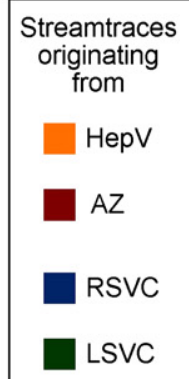

FIGURE 4. Flow patterns and HFD for selected options for patient B2, under a global CO distribution of 50/50 RPA/LPA. Options include (A) an intraatrial connected opposite to the RSVC, (B) a Y-graft, (C) an H-connection using an extracardiac to the LSVC and an HepV-to-AZ shunt, and (D) an AZto-HepV shunt combined with a Y-graft. Orientation axis: $\mathrm{S}=$ superior; $\mathrm{I}=$ inferior; $\mathrm{L}=$ left; $\mathrm{R}=$ right. AZ, Azygous; HepV, hepatic veins; $L P A$, left pulmonary artery; $R P A$, right pulmonary artery; $R S V C$, right superior vena cava; $L S V C$, left superior vena cava. 
for an easy connection of the hepatic baffle; (2) the hepatic flow rate was not too low, accounting for approximately $20 \%$ of the total systemic venous return; (3) and, most important, the right superior venous return (in this case, the RSVC) was almost exactly equal to the left superior venous inflows (in this case, the AZ + LSVC). This last condition should be considered with care, especially when optimizing the TCPC for younger patients. Indeed, having equal left and right superior venous return at the time of TCPC surgery in younger patients does not warrant that this balance will hold as the patient grows. AZ, hepatic, RSVC, and LSVC flow rates will most likely evolve with age, which might alter the balance of the left and right superior inflows and lead to a severely imbalanced state, such as in patient B2.

Alternate options of interest are the Y- or H-graft configurations that seek to divide the hepatic flow between a left and a right branch. A particularity of patients with bilateral SVCs is that, as pointed out for patient B1, HepV-to-AZ shunts have the same effect as an offset toward the PA closest to the AZ. An H-connection for these patients thus circumvents the difficulties encountered with Y-grafts, allowing the hepatic blood flow to reach the PA farthest away from the HepV without the spatial constraints imposed by the aortic arch. Finally, in patients in whom the hepatic flow is low or the majority of flow comes in from the side where the AZ is connected (as was the case for patient B2), diverting the AZ flow from the Kawashima into the hepatic baffle and then optimizing the baffle design may be a suitable approach.

\section{DISCUSSION}

We presented the surgical planning studies conducted by our group for patients with a single ventricle with an interrupted IVC, reviewing the preoperative performance, a range of reoperation scenarios, and the recommended surgical strategies. Although postoperative magnetic resonance imaging data are lacking, the recommended approaches led to increased oxygen saturations (from $72 \% \pm 4 \%$ preoperatively to $90 \% \pm 7 \%$ postoperatively), leading to improved HFD and successful surgical implementation. Contrasting the recommended reoperative scenarios with the preoperative anatomies that were determined by the surgeon on the basis of anatomic considerations alone, this study demonstrates how clinical magnetic resonance imaging and new virtual surgery environments can benefit the clinical community, especially for patients with a single ventricle with an interrupted IVC.

This systematic surgical planning investigation provides a set of guidelines to be followed when treating patients with an interrupted IVC to optimize HFD and ultimately minimize the chance of PAVM. Because of the low flow rate coming through the TCPC baffle, HFD was sensitive to the offset between the hepatic baffle and the "flow center" of the superior venous returns, and even a small offset toward the LPA or RPA led to a highly preferential HFD to the associated lung. For patients with an interrupted IVC and a single SVC, "classic" intra-atrial or extracardiac approaches thus proved dangerous because of the difficulty in identifying the anastomosis location to minimize the offset between the hepatic baffle and the "flow center" of the superior venous returns, especially because this location depends on the complex interaction of the AZ, IV, and SVC flows. This fact alone underscores the value of the present techniques in regard to preoperative surgery planning. In the absence of a patient-specific offset optimization, Ygrafts or HepV-to-AZ shunts appeared as 2 attractive alternatives. HepV-to-AZ shunts ensured a thorough mixing of all venous returns, although at higher energy expenses. When successful, a Y-graft allowed for both energy and HFD efficiency, but implementation was in practice hindered by the aorta. This technique is still being explored and, with further refinement, may become a viable and successful option for a larger patient pool.

For patients with bilateral SVC, results depended on the balance between the left and right superior inflows. When those were approximately equal, optimal results were obtained by centrally connecting the hepatic baffle between the SVCs, along the mid-PA segment. Alternate options included $\mathrm{H}$-connections or routing the AZ flow into the hepatic baffle and subsequently optimizing the baffle design.

\section{CONCLUSIONS}

All the cases reported demonstrate a strong sensitivity of the optimal baffle design on the distribution of the systemic venous return between the different vessels and the resultant flow interaction. Determining the best suited option for a given patient thus requires a detailed analysis of not only anatomy but also flow distributions, keeping in mind that the measured flow rates may evolve as the patient ages. Future work should thus involve a detailed error analysis to determine the sensitivity of a potential option to variations in the systemic flow rates.

\section{References}

1. Duncan BW, Desai S. Pulmonary arteriovenous malformations after cavopulmonary anastomosis. Ann Thorac Surg. 2003;76:1759-66.

2. Pandurangi UM, Shah MJ, Murali R, Cherian KM. Rapid onset of pulmonary arteriovenous malformations after cavopulmonary anastomosis. Ann Thorac Surg. 1999;68:237-9.

3. Pike NA, Vricella LA, Feinstein JA, Black MD, Reitz BA. Regression of severe pulmonary arteriovenous malformations after Fontan revision and "hepatic factor" rerouting. Ann Thorac Surg. 2004;78:697-9.

4. Shinohara T, Yokoyama T. Pulmonary arteriovenous malformation in patients with total cavopulmonary shunt: what role does lack of hepatic venous blood flow to the lungs play? Pediatr Cardiol. 2001;22:343-6.

5. Shah MJ, Rychik J, Fogel MA, Murphy JD, Jacobs ML. Pulmonary AV malformations after superior cavopulmonary connection: resolution after inclusion of hepatic veins in the pulmonary circulation. Ann Thorac Surg. 1997;63:960-3.

6. Sundareswaran KS, de Zelicourt D, Sharma S, Kanter KR, Spray TL, Rossignac J, et al. Correction of pulmonary arteriovenous malformation using image-based surgical planning. J Am Coll Cardiol Img. 2009;2:1024-30. 
7. Frakes DH, Conrad CP, Healy TM, Monaco JW, Fogel M, Sharma S, et al. Application of an adaptive control grid interpolation technique to morphological vascular reconstruction. IEEE Trans Biomed Eng. 2003;50:197-206.

8. Pekkan K, Whited B, Kanter K, Sharma S, de Zelicourt D, Sundareswaran K, et al. Patient-specific surgical planning and hemodynamic computational fluid dynam- ics optimization through free-form haptic anatomy editing tool (SURGEM). Med Biol Eng Comput. 2008;46:1139-52.

9. de Zélicourt D, Ge L, Wang C, Sotiropoulos F, Gilmanov A, Yoganathan A. Flow simulations in arbitrarily complex cardiovascular anatomies-an unstructured Cartesian grid approach. Comput Fluids. 2009;38:1749-62.

Access to The Journal of Thoracic and Cardiovascular Surgery Online is reserved for print subscribers!

Full-text access to The Journal of Thoracic and Cardiovascular Surgery Online is available for all print subscribers. To activate your individual online subscription, please visit The Journal of Thoracic and Cardiovascular Surgery Online, point your browser to http://www.mosby.com/itcvs, follow the prompts to activate your online access, and follow the instructions. To activate your account, you will need your subscriber account number, which you can find on your mailing label (note: the number of digits in your subscriber account number varies from 6 to 10). See the example below in which the subscriber account number has been circled:

\section{Sample mailing label}

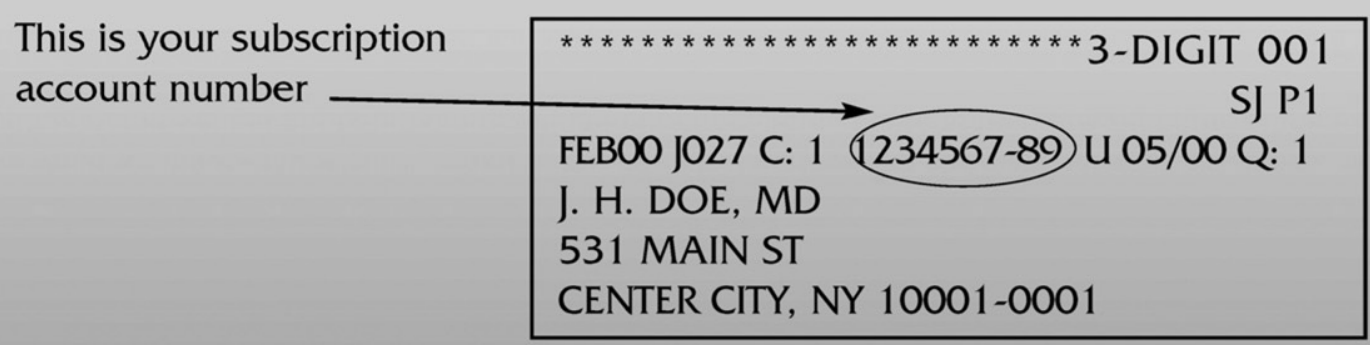

Personal subscriptions to The Journal of Thoracic and Cardiovascular Surgery Online are for individual use only and may not be transferred. Use of The Journal of Thoracic and Cardiovascular Surgery Online is subject to agreement to the terms and conditions as indicated online. 